\title{
Dynamics of collapsing and exploding Bose-Einstein condensates
}

\author{
Elizabeth A. Donley, Neil R. Claussen, Simon L. Cornish, Jacob L. Roberts, Eric A. Cornell and Carl E. Wieman \\ JILA, National Institute of Standards and Technology and the University of Colorado, \\ and the Department of Physics, University of Colorado, Boulder, Colorado 80309-0440
}

(Dated: October 31, 2018)

\begin{abstract}
We explored the dynamics of how a Bose-Einstein condensate collapses and subsequently explodes when the balance of forces governing the size and shape of the condensate is suddenly altered. A condensate's equilibrium size and shape is strongly affected by the inter-atomic interactions. Our ability to induce a collapse by switching the interactions from repulsive to attractive by tuning an externally-applied magnetic field yields a wealth of detailed information on the violent collapse process. We observe anisotropic atom bursts that explode from the condensate, atoms leaving the condensate in undetected forms, spikes appearing in the condensate wave function, and oscillating remnant condensates that survive the collapse. These all have curious dependencies on time, the strength of the interaction, and the number of condensate atoms. Although ours would seem to be a simple well-characterized system, our measurements reveal many interesting phenomena that challenge theoretical models.
\end{abstract}

Although the density of the atoms in an atomic BoseEinstein condensate (BEC) is typically five orders of magnitude lower than the density of air, the inter-atomic interactions greatly affect a wide variety of BEC properties. These include static properties like the BEC size and shape and the condensate stability, and dynamic properties like the collective excitation spectrum and soliton and vortex behavior. Since all of these properties are sensitive to the inter-atomic interactions, they can be quite dramatically affected by tuning the interaction strength and sign.

The vast majority of BEC physics is well described by mean-field theoryl, in which the strength of the interactions depends on the atom density and on one additional parameter called the $s$-wave scattering length $a$. $a$ is determined by the atomic species. When $a>0$, the interactions are repulsive. In contrast, when $a<0$ the interactions are attractive and a BEC tends to contract to minimize its overall energy. In a harmonic trap, the contraction competes with the kinetic zero-point energy, which tends to spread out the condensate. For a strong enough attractive interaction, there is not enough kinetic energy to stabilize the BEC and it is expected to implode. A BEC can avoid implosion only as long as the pumber of atoms $N_{0}$ is less than a critical value given by

$$
N_{c r}=k a_{h o} /|a|,
$$

where dimensionless constant $k$ is called the stability coefficient. The precise value of $k$ depends on the aspect ratio of the magnetic trap 3 . $a_{h o}$ is the harmonic oscillator length, which sets the size of the condensate in the ideal-gas $(a=0)$ limit.

Under most circumstances, $a$ is insensitive to external fields. This is different in the vicinity of a so-called Feshbach resonance, where $a$ can be tuned over a huge rapore by adjusting the externally applied magnetic field此. This has been demenstrated in recent years with cold

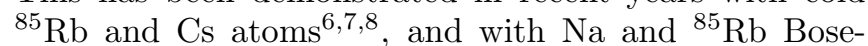
Einstein condensates 10 . For ${ }^{85} \mathrm{Rb}$ atoms, $a$ is usually negative, but a Feshbach resonance at $\sim 155 \mathrm{G}$ allows us to tune $a$ by orders of magnitude and even change its sign. This gives us the ability to create stable ${ }^{85} \mathrm{Rb}$ Bose-Einstein condensates 10 and adjust the inter-atomic interactions. We recently used this flexibility to verify the functional form of equation (11) and to measure the stability coefficient to be $k=0.46(6)^{11}$.

In this article, we study the dynamical response ("the collapse") of an initially stable BEC to a sudden shift of the scattering length to a value more negative than the critical value $a_{c r}=-k a_{h o} / N_{0}$. We have observed many features of the surprisingly complex collapse process, including the energies and energy anisotropies of atoms that burst from the condensate, the time scale for the onset of this burst, the rates for losing atoms, spikes in the wave function that form during collapse, and the size of the remnant BEC that survives the collapse. The unprecedented level of control provided by tuning $a$ has allowed us to see how all of these quantities depend on the magnitude of $a$, the initial number and density of condensate atoms, and the initial spatial size and shape of the BEC before the transition to instability.

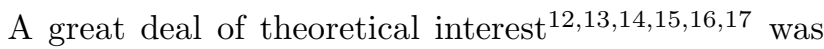
generated by $\mathrm{BEC}$ experiments in ${ }^{7} \mathrm{Li} 18$, for which the scattering length is also negative and collapse events are also observedined. The ${ }^{7} \mathrm{Li}$ experiments do not employ a Feshbach resonance, so $a$ is fixed. This restricts experimentation to the regime where the initial number of condensate atoms is less than or equal to $N_{c r}$, and the collapse is driven by a stochastic process. In addition, studies of collapse dynamics in ${ }^{7} \mathrm{Li}$ are complicated by a large thermal component. Our ability to tune the scattering length, and most notably explore the regime where the initial condensate is "pure" (near $T=0$ ) and the number $N_{0}$ is much larger than $N_{c r}$, allows us to explore the dynamics and compare it with theory in far more detail.

Experimental techniques. The procedure for producing stable ${ }^{85} \mathrm{Bb}$ condensates has been described in detail elsewhere 10 . A standard double magneto-optical trap (MOT) system² was used to collect a cold sample 
of ${ }^{85} \mathrm{Rb}$ atoms in a low-pressure chamber. Once sufficient atoms had accumulated in the low-pressure MOT, the atoms were loaded into a cylindrically symmetric cigar-shaped magnetic trap with trap the frequencies $\nu_{\text {radial }}=17.5 \mathrm{~Hz}$ and $\nu_{\text {axial }}=6.8 \mathrm{~Hz}$. Radio-frequency evaporation was then used to cool the sample to $\sim 3 \mathrm{nK}$ to form pure condensates containing $>90 \%$ of the sample atoms. The final stages of evaporation were performed at $162 \mathrm{G}$ where the scattering length is positive and stable condensates of up to 15,000 atoms could be formed. After evaporative cooling, the magnetic field was ramped adiabatically to $166 \mathrm{G}$ (except where noted), where $a=0$. This provided a well-defined initial condition with the BEC taking on the size and shape of the harmonic oscillator ground state.

We could then adjust the mean-field interactions within the BEC to a variety of values on time scales as short as $0.1 \mathrm{~ms}$. The obvious manipulation was to jump to some value of $a<a_{c r}$ to trigger a collapse, but the tunability of $a$ also greatly aided in imaging the sample. Usually the condensate size was below the resolution limit of our imaging system ( $7 \mu \mathrm{m}$ FWHM). However, we could ramp the scattering length to large positive values and use the repulsive inter-atomic interactions to expand the BEC before imaging, thus obtaining information on the pre-expansion condensate shape and number. A typical $a(t)$ sequence is shown in Fig. 17a. We have used a variety of such sequences to explore many aspects of the collapse and enhance the visibility of particular components of the sample.

Condensate contraction and atom loss. When the scattering length is jumped to a value $a_{\text {collapse }}<$ $a_{c r}$, a condensate's kinetic energy no longer provides a sufficient barrier against collapse. As described in ref. 13, during collapse one might expect a BEC to contract until losses from density-dependent inelastic collision 22 would effectively stop the contraction. This contraction would roughly take place on the time scale of a trap oscillation, and the density would sharply increase after $T_{\text {rad }} / 4 \simeq$ $14 \mathrm{~ms}$, where $T_{\text {rad }}$ is the radial trap period. How does this picture compare to what we have actually seen?

A plot of the condensate number $N$ vs $\tau_{\text {evolve }}$ for $a_{\text {collapse }}=-30 a_{0}$ and $a_{\text {init }}=+7 a_{0}$ is presented in Fig. 1]b. $N$ was constant for some time after the jump until atom loss suddenly began at $t_{\text {collapse. }}$ After the jump the condensate was smaller than our resolution limit, so we could not observe the contraction directly. But we observed that the post-expansion condensate widths changed very little with time $\tau_{\text {evolve }}$ before $t_{\text {collapse }}$. From this we infer that the bulk BEC did not contract dramatically before loss began. We modelled the contraction by putting the $a(t)$ sequence that we used for the experiment into the equations of ref. 24 for the approximate evolution of the BEC shape. The model predicted that by $t_{\text {collapse }}$, the radial and axial widths had contracted by $\sim 20 \%$ and $\sim 4 \%$, respectively, from their initial values. This contraction only corresponds to a $50 \%$ increase in the average density to $2.5 \times 10^{13} / \mathrm{cm}^{3}$. Using the decay constants from ref. 22, this density gives an atom loss rate, $\tau_{\text {decay }}$, that is far smaller than what we observe and does not have the observed sudden onset.

For the data in Fig. 1]b and most other data presented below, we jumped to $a_{\text {quench }}=0$ in $0.1 \mathrm{~ms}$ after a time $\tau_{\text {evolve }}$ at $a_{\text {collapse }}$. We believe that the loss immediately stopped after the jump. This interpretation is based on the surprising observation that the quantitative details of curves such as that shown in Fig. 11b did not depend on whether the collapse was terminated by a jump to $a_{\text {quench }}=0$ or $a_{\text {quench }}=250 a_{0}$.

We have measured loss curves like that in Fig. 目 for many different values of $a_{\text {collapse }}$. The collapse time versus $a_{\text {collapse }}$ for $N_{0}=6000$ presented in Fig. 22 shows the strong dependence on $a_{\text {collapse }}$. Reducing the initial density by a factor of $\sim 4$ (with a corresponding increase in volume) by setting $a_{\text {init }}=+89 a_{0}$ for one value of $a_{\text {collapse }}\left(-15 a_{0}\right)$, increased $t_{\text {collapse }}$ by $\sim 3$.

The atom loss time constant $\tau_{\text {decay }}$ depended only weakly on $a_{\text {collapse }}$ and $N_{0}$. For the range of $a_{\text {collapse }}$ shown in Fig. 2, $\tau_{\text {decay }}$ did not depend on $a_{\text {collapse }}$ or $N_{0}$ outside of the experimental noise $(\sim 20 \%)$. On average, $\tau_{\text {decay }}$ was $2.8(1) \mathrm{ms}$. For the very negative value of $a_{\text {collapse }} \simeq-250 a_{0}$, however, $\tau_{\text {decay }}$ did decrease to $1.8 \mathrm{~ms}$ for $N_{0}=6,000$ and $1.2 \mathrm{~ms}$ for $N_{0}=15,000$.

Burst atoms. As indicated by Fig. 1 b, atoms leave the BEC during the collapse. There are at least two components to the expelled atoms. One component (the "missing atoms") is not detected. The other component emerges as a burst of detectable spin-polarized atoms with energies much greater than the initial condensate's energy but much less than the magnetic trap depth. The burst-energy dependencies on $a_{\text {collapse }}$ and $N_{0}$ are complex, but since they will provide a stringent test of collapse theories, we present them in detail.

The angular kinetic-energy distribution with which the burst atoms are expelled from the condensate can most accurately be measured by observing their harmonic oscillations in the trap, as illustrated in Fig. 3a. For example, half of a radial period after the expulsion $\left(T_{\text {rad }} / 2\right)$, all atoms return to their initial radial positions. Well before or well after this "radial focus", the burst cloud is too dilute to be observed. Fortunately, at the radial focus, oscillations along the axial trap axis are near their outer turning points and the axial energy can be found from the length of the stripe of atoms along the axial axis. The radial energy can be found with the same procedure for an axial focus. The sharpness of the focus also provides information on the time extent of the burst.

Figure 3b shows an image of a radial focus. The size scales for the burst focus and the remnant were well separated since the latter was not expanded before imaging. Figure 3c shows cross sections of the burst focus and fits to the burst and the thermal cloud. The burst energy distributions were well fit by Gaussians characterized by a temperature that was usually different for the two trap directions. The burst energy fluctuated from shot to shot by up to a factor of 2 for a given $a_{\text {collapse }}$. This varia- 
tion is far larger than the measurement uncertainty or the variation in initial number (both $\sim 10 \%$ ), and its source is unknown. (We also discuss observed structural variability when we present the jet measurements below.)

Although the burst energies varied from shot to shot, the average value was well-defined and showed trends far larger than the variation. The axial and radial burst

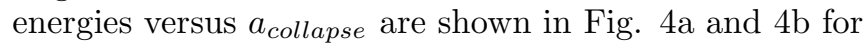
$N_{0}=6,000$ and 15,000 , respectively. The burst-energy anisotropy shown in Fig. $4 \mathrm{c}$ c depended on $N_{0}, a_{\text {collapse }}$, and $a_{\text {init }}$.

When we interrupted the collapse with a jump back to $a=0$ as discussed above, we also interrupted the growth of the burst. The "interrupted" burst atoms still refocused after sitting at $a=0$ for the requisite half trap period. The energy of the atoms in the interrupted bursts appeared to be the same, but the number of atoms was smaller. By changing the time at which the collapse was interrupted we could measure the time dependence of the creation of burst atoms. For the conditions of Fig. 1 $1 \mathrm{~b}$, the number of burst atoms $N_{\text {burst }}$ grew with $\tau_{\text {evolve }}$ with a time constant of $1.2 \mathrm{~ms}$ starting at $3.5 \mathrm{~ms}$ and reaching an asymptotic final number of $\sim 2500$ for all times $\geq 7 \mathrm{~ms}$. $N_{\text {burst }}$ varied randomly by $\sim 20 \%$ for the data in Fig. 4 , but on average the fraction of atoms going into the burst was about $20 \%$ of $N_{0}$ and did not depend on $a_{\text {collapse }}$ or $N_{0}$.

Remnant Condensate. After a collapse, a "remnant" condensate containing a fraction of the atoms survived with nearly constant number for more than $1 \mathrm{sec}-$ ond and oscillated in a highly excited collective state with the two lowest modes with $\nu \simeq 2 \nu_{\text {axial }}$ and $\nu \simeq 2 \nu_{\text {radial }}$ being predominantly excited. (The measured frequencies were $\nu=13.6(6) \mathrm{Hz}$ and $\nu=33.4(3) \mathrm{Hz}$.) To find the oscillation frequencies, the widths of the condensate were measured as a function of time spent at $a_{\text {collapse }}$.

The number of atoms in the remnant depended on $a_{\text {collapse }}$ and $N_{0}$, and in general was not limited by the critical number, $N_{c r}$. The stability condition in equation (11) determined the collapse point but it did not constrain $N_{\text {remnant }}$. A fixed fraction of $N_{0}$ went into the remnant independent of $N_{0}$, so that smaller condensates often ended up with $N_{\text {remnant }}<N_{c r}$, but larger condensates rarely did. The fraction of atoms that went into the remnant decreased with $\left|a_{\text {collapse }}\right|$, and was $\sim 40 \%$ for $\left|a_{\text {collapse }}\right|<10 a_{0}$ and $\sim 50 \%$ for $\left|a_{\text {collapse }}\right|>1 \rho a_{0}$. We do not think that the surface-wave excitations 25 are responsible for stabilizing the remnant since we excite large-amplitude breathing modes. For $N_{0}=6,000$ and $\left|a_{\text {collapse }}\right|<10 a_{0}$, more atoms were lost than the number required to lower $N_{\text {remnant }}$ to below $N_{c r}$.

Since $N_{\text {burst }}$ was independent of $a_{\text {collapse }}$ but $N_{\text {remnant }}$ decreased with $\left|a_{\text {collapse }}\right|$, the number of missing atoms increased with $\left|a_{\text {collapse }}\right|$. Interestingly, the number of missing atoms also increased with $N_{0}$, but the fraction of missing atoms versus $a_{\text {collapse }}$ was equal for $N_{0}=6,000$ and $N_{0}=15,000$ and was $\sim 40 \%$ for $\left|a_{\text {collapse }}\right|<10 a_{0}$ and $\sim 70 \%$ for $\left|a_{\text {collapse }}\right| \geq 100 a_{0}$. The missing atoms were presumably either expelled from the condensate at such high energies that we could not detect them $(>20 \mu \mathrm{K})$, or they were transferred to untrapped atomic states or undetected molecules.

Jet formation. Under very specific experimental conditions, we observed streams of atoms with highly anisotropic velocities emerging from the collapsing condensates. These "jets" are distinguished from the "burst" in that the jets have much lower kinetic energy (on the order of a few nanokelvin), in that their velocity is nearly purely radial, and in that they appear only when the collapse is interrupted (i.e., by jumping to $a_{\text {quench }}=0$ ) during the period of number loss. Collapse processes that were allowed to evolve to completion (until $N \simeq N_{\text {remnant }}$ ) were not observed to emit jets. Examples are shown in Fig. 5 for different $\tau_{\text {evolve }}$ for the conditions of Fig. 1 b. The jet size and shape varied from image to image even when all conditions were unchanged, and as many as three jets were sometimes observed to be emitted from the collapse of a single condensate. The jets were also not always symmetric about the condensate axis.

We believe that these jets are manifestations of local "spikes" in the condensate density that form during the collapse and expand when the balance of forces is changed by quenching the collapse. We can estimate the size of the spikes using the uncertainty principle. After a jump to $a_{\text {quench }}=0$, the kinetic energy of the atoms in the resulting jet is equal to the confinement energy that the spike had prior to quenching the collapse, i.e., $\frac{1}{2} m v^{2}=\frac{\hbar^{2}}{4 m \sigma^{2}}$, where $\sigma$ is the width of the spike in the wave function. The anisotropy of the jets indicates that the spikes from which they originated were also highly anisotropic, being narrower in the radial direction. From the widths and the number of atoms in the jets, we can estimate the density in the spikes. Plots of the number of jet atoms and the inferred density in the spikes versus $\tau_{\text {evolve }}$ are presented in Fig. 6. The jets exhibited variability in energy and number that was larger than the $\sim 10 \%$ measurement noise.

Overview of the curpent thepretical models. Several theoretical papers12]13,14,15.16.17 have considered the problem of collapse of a BEC when the number of atoms exceeds the critical number. These treatments all use a mean-field approach and describe the condensate dynamics usipg the Gross-Pitaevskii (GP) equation. In most cases 12,13 . 15.16, the loss mechapism is three body recombination, but Duine and Stoo@ 17 propose the loss arises from a new elastic scattering process. In both cases the loss is density dependent and so the loss rate is quite sensitive to the dynamics of the shape of the condensate. Since a full three-dimensional anisotropic timedependent solution to the GP equation is very difficult, these calculations have used various approximations to calculate the time evolution of the condensate shape. Kagan, Muryshev, and Shlyapnikov 13 numerically integrate the GP equation for the case of an isotropic trap and large values of the three body recombination coefficient. In this regime, the condensate smoothly contracts in a 
single, collective collapse. Saito and Ueda 15.16 perform a similar numerical solution for the isotropic case but with smaller values of $K_{3}$ and observe localized spikes to form in the wave function during collapse. Duine and Stooft 7 model the dynamics for the anisotropic case, but use a Gaussian approximation rather than an exact numerical solution.

These calculations have all been carried out over a certain range of parameters, but none have been done for the specific range of parameters that correspond to our experimental situations. None of the predictions in these papers match our measurements except for the general feature that atoms are lost from the condensate. Also we see several phenomena that are not discussed in these papers. Whether this lack of agreement is due to the fact that these calculations do not scale to our experimental situation or do not contain the proper physics remains to be seen.

Theoretical Challenges. Collapsing ${ }^{85} \mathrm{Rb}$ condensates present a rather simple system with quite dramatic behavior. This behavior might provide a rigorous test of mean-field theory when it is applied to our experimental conditions. Some of our particularly puzzling results are:

- The decay constant $\tau_{\text {decay }}$ is independent of both
$N_{0}$ and $\left|a_{\text {collapse }}\right|$ for $\left|a_{\text {collapse }}\right|<100 a_{0}$, and only weakly depends on these quantities for larger $\left|a_{\text {collapse }}\right|$.

- The burst energy per atom dramatically increases with initial condensate number.

- The number of burst atoms is constant versus $a_{\text {collapse. }}$.

- The number of cold remnant BEC atoms surviving the violent collapse varies between much less and much more than $N_{c r}$, depending on $N_{0}$ and $a_{\text {collapse }}$, but the fraction of remnant atoms, burst atoms, and missing atoms are independent of $N_{0}$.

Outlook. From the experimental point of view there remain questions to be answered. For one, is the burst coherent? It may be possible to answer this by generating a sequence of "half bursts" and see if they interfere. For another, where do the missing atoms go? If molecules and/or relatively high-energy atoms are being created, can we detect them?

It is clear that adjustable interactions opens up a fascinating new regime for BEC studies.
* Quantum Physics Division, National Institute of Standards and Technology.

1 Dalfovo, F., Giorgini, S., Pitaevskii, L. P., \& Stringari, S. Theory of Bose-Einstein condensation in trapped gases. Rev. Mod. Phys. 71, 463-512 (1999).

${ }^{2}$ Ruprecht, P. A., Holland, M. J., Burnett, K., \& Edwards, M. Time-dependent solution of the nonlinear Schrödinger equation for Bose-condensed trapped neutral atoms. Phys. Rev. A 51, 4704-4711 (1995).

3 Gammal, A., Frederico, T., \& Tomio, L. Critical number of atoms for attractive Bose-Einstein condensates with cylindrically symmetrical traps. Phys. Rev. A (submitted.) (2001).

4 Tiesinga, E., Moerdijk, A. J., Verhaar, B. J., \& Stoof, H. T. C. Conditions for Bose-Einstein condensation in magnetically trapped atomic cesium. Phys. Rev. A 46, R1167 (1992).

5 Tiesinga, E., Verhaar, B. J., \& Stoof, H. T. C. Threshold and resonance phenomena in ultracold ground-state collisions. Phys. Rev. A 47, 4114-4122 (1993).

${ }^{6}$ Courteille, Ph., Freeland, R. S., Heinzen, D. J., van Abeelen, F. A., \& Verhaar, B. J. Observation of a Feshbach resonance in cold atom scattering. Phys. Rev. Lett. 81, 69-72 (1998).

7 Roberts, J. L., et al. Resonant magnetic field control of elastic scattering in cold ${ }^{85}$ Rb. Phys. Rev. Lett. 81, 51095112 (1998).

${ }^{8}$ Vuletić, V., Kerman, A. J., Chin, C., \& Chu, S. Observation of low-field Feshbach resonances in collisions of cesium atoms. Phys. Rev. Lett. 82, 1406-1409 (1999).

9 Inouye, S. et al. Observation of Feshbach resonances in a Bose-Einstein condensate. Nature 392, 151-154 (1998).
${ }^{10}$ Cornish, S. L., Claussen, N. R., Roberts, J. L., Cornell, E. A., \& Wieman, C. E. Stable ${ }^{85}$ Rb Bose-Einstein condensates with widely tunable interactions. Phys. Rev. Lett. 85, 1795-1798 (2000).

11 Roberts, J. L., et al. Controlled collapse of a Bose-Einstein condensate. Phys. Rev. Lett. 86, 4211-4214 (2001).

12 Kagan, Y., Surkov, E. L., \& Shlyapnikov, G. V. Evolution and global collapse of trapped Bose condensates under variations of the scattering length. Phys. Rev. Lett. 79, 2604-2607 (1997).

13 Kagan, Y., Muryshev, A. E., \& Shlyapnikov, G. V. Collapse and Bose-Einstein condensation in a trapped Bose Gas with negative scattering length. Phys. Rev. Lett. 81, 933-937 (1998).

14 Ueda, M. \& Huang, K. Fate of a Bose-Einstein condensate with an attractive interaction. Phys. Rev. A 60, 3317-3320 (1999).

15 Saito, H. \& Ueda, M. Power laws and collapsing dynamics of a trapped Bose-Einstein condensate with attractive interactions. Phys. Rev. A 63, 043601-7 (2001).

16 Saito, H. \& Ueda, M. Intermittent implosion and pattern formation of trapped Bose-Einstein condensates with an attractive interaction. Phys. Rev. Lett. 86, 1406-1409 (2001).

17 Duine, R. A. \& Stoof, H. T. C. Explosion of a collapsing Bose-Einstein condensate. Phys. Rev. Lett. 86, 2204-2207 (2001).

18 Bradley, C. C., Sackett, C. A. \& Hulet, R. G. Bose-Einstein condensation of Lithium: Observation of Limited condensate number. Phys. Rev. Lett. 78, 985-988 (1997).

19 Sackett, C. A., Gerton, J. M., Welling, M., \& Hulet, R. G. Measurements of collective collapse in a Bose-Einstein con- 
densate with attractive interactions. Phys. Rev. Lett. 82, 876-879 (1999).

${ }^{20}$ Gerton, J. M., Strekalov, D., Prodan, I., \& Hulet, R. G. Direct observation of growth and collapse of a Bose-Einstein condensate with attractive interactions. Nature 408, 692695 (2001).

21 Myatt, C. J., Newbury, N. R., Ghrist, R. W., Loutzenhiser, S., \& Wieman, C. E. Multiply loaded magneto-optical trap. Opt. Lett. 21, 290-292 (1996).

${ }^{22}$ Roberts, J. L., Claussen, N. R., Cornish, S. L., \& Wieman, C. E. Magnetic field dependence of ultracold inelastic collisions near a Feshbach resonance. Phys. Rev. Lett. 85, 728-731 (2000).

23 Stenger, J., et al.. Strongly enhanced inelastic collisions in a Bose-Einstein condensate near Feshbach resonances. Phys. Rev. Lett. 82, 2422-2425 (1999).

24 Pérez-García, V. M., Michinel, H., Cirac, J. I., Lewen- stein, M., \& Zoller, P. Dynamics of Bose-Einstein condensates: Variational solutions of the Gross-Pitaevskii equations. Phys. Rev. A 56, 1424-1432 (1997).

25 Pattanayak, A. K., Gammal, A., Sackett, C. A., \& Hulet, R. G. Stabilizing an attractive Bose-Einstein condensate by driving a surface collective mode. Phys. Rev. A 63, 033604-4 (2001).

ACKNOWLEDGMENTS. We extend our thanks to Sarah Thompson for laboratory assistance and to Stephan Dürr, Gora Shlyapnikov, Henk Stoof, Murray Holland, Masahito Ueda, and Rembert Duine for helpful discussions. This research has been supported by the ONR, NSF, ARO-MURI, and NIST. S. L. C. acknowledges the support of a Lindemann Fellowship. 
FIG. 1: A example of a ramp applied to the scattering length, and a plot of the condensate number versus time after a jump to a negative scattering length. a. A typical $a(t)$ sequence. $a_{0}=0.529 \AA$ is the Bohr radius. The scattering length is jumped at $t=0$ in $0.1 \mathrm{~ms}$ from $a_{\text {init }}$ to $a_{\text {collapse, }}$, where the BEC evolves for a time $\tau_{\text {evolve }}$. The field is carefully controlled so that magnetic-field noise translates into fluctuations in $a_{\text {collapse }}$ on the order of $\sim 0.1 a_{0}$ in magnitude. The collapse is then interrupted with a jump to $a_{\text {quench }}$, and the field is ramped in $5 \mathrm{~ms}$ to a large positive scattering length which makes the BEC expand. After $7.5 \mathrm{~ms}$ of additional expansion, the trap is turned off in $0.1 \mathrm{~ms}$ and $1.8 \mathrm{~ms}$ later the density distribution is probed using destructive absorption imaging with a $40 \mu$ s laser pulse (indicated by the vertical bar). The increase in $a$ from $a_{\text {collapse }}$ to $a_{\text {expand }}$ is far too rapid to allow for the BEC to expand adiabatically. On the contrary, the smaller the BEC before expansion, the larger the cloud at the moment of imaging. Thus we can readily infer the relative size of the bulk of the BEC just prior to the jump to $a_{\text {quench }}$. The density of the expanded BEC is splow that the rapid transit of the Feshbach resonance pole 23 during the trap turn-off and the subsequent time spent at $B=0\left(a=-400 a_{0}\right)$ both have a negligible effect. $b$. The number of atoms remaining in the BEC versus $\tau_{\text {evolve }}$ at $a_{\text {collapse }}=-30 a_{0}$. We observed a delayed and abrupt onset of loss. The solid line is a fit to an exponential with a best-fit value of $t_{\text {collapse }} \simeq 3.7(5) \mathrm{ms}$ for the delay.

FIG. 2: The collapse time versus $a_{\text {collapse }}$ for 6000 -atom condensates. The vertical line indicates $a_{c r}$ for $N_{0}=6,000$. The data were acquired with $a_{\text {init }}=a_{\text {quench }}=0$ (to within $\left.\sim 2 a_{0}\right)$.

FIG. 3: A burst focus. a. Conceptual illustration of a radial burst focus. b. An image of a radial burst focus taken $33.5 \mathrm{~ms}$ after a jump from $a_{\text {init }}=0$ to $-30 a_{0}$ for $N_{0}=15,000$. $T_{\text {rad }} / 2=28.6 \mathrm{~ms}$, which indicates that the burst occurred 4.9(5) ms after the jump. The axial energy distribution for this burst corresponded to an effective temperature of $62 \mathrm{nK}$. The image is $60 \times 310 \mu \mathrm{m}$. c. Radially averaged cross section of $\mathrm{b}$ with a Gaussian fit to the burst energy distribution. The central $100 \mu \mathrm{m}$ were excluded from the fit to avoid distortion in the fit due to the condensate remnant $(\sigma=9 \mu \mathrm{m})$ and the thermal cloud $(\sigma=17 \mu \mathrm{m})$. The latter is present in the precollapse sample due to the finite temperature and appears to be unaffected by the collapse. The dashed line indicates the fit to this initial thermal component. Note the offset between the centers of the burst and the remnant. This offset varies from shot to shot by an amount comparable to the offset shown. 
FIG. 4: Burst energies and energy anisotropies. a. and b. The axial and radial burst energies versus $a_{\text {collapse }}$ for $N_{0} \simeq 6,000$ and $N_{0} \simeq 15,000$, respectively. On average, ten burst focuses were measured for each trap direction at each value of $a_{\text {collapse }}$ studied. The energies were higher for the larger- $N_{0}$ condensates over the full range of $a_{\text {collapse }}$ studied. The vertical and horizontal error bars indicate the standard error of the measurements and the uncertainty in $a_{\text {collapse }}$ arising from the magnetic-field calibration, respectively. For several of the points, the uncertainties are smaller than the symbol size and the error bars are not visible. c. The ratio of the radial to the axial energies, which is a measure of the burst anisotropy. For values of $\left|a_{\text {collapse }}\right|$ just past $a_{c r}$, the burst was isotropic for both $N_{0} \simeq 6,000$ and $N_{0} \simeq 15,000$. At larger values of $\left|a_{\text {collapse }}\right|$, larger- $N_{0}$ condensates gave rise to stronger anisotropies. For $N_{0}=6000, \lambda$ was 1.6, and for $N_{0}=15,000, \lambda$ was 2 . When instead we started at $a_{\text {init }}=$ $+100 a_{0}$, the BEC was initially more anisotropic $(\lambda=2.4)$, but the burst became more isotropic, with $E_{a x}$ going up by $\sim 40 \%$ and $E_{\text {rad }}$ dropping by $\sim 60 \%$ for $N_{0}=15,000$ and $a_{\text {collapse }}=-100 a_{0}$.

FIG. 5: Jet images for a series of $\tau_{\text {evolve }}$ values for the conditions of Fig. Ab. The evolution times were 2, 3, 4, 6, 8 , and $10 \mathrm{~ms}$ (from a to f). Each image is $150 \times 255 \mu \mathrm{m}$. The bar indicates the optical depth scale. An expansion to $a_{\text {expand }}=+250 a_{0}$ was applied, so the jets are longer than for the quantitative measurements explained in the text. The jets were longest (i.e., most energetic) and contained the most atoms at values of $\tau_{\text {evolve }}$ for which the slope of the loss curve (Fig. 1 $1 \mathrm{~b}$ ) was greatest. A tiny jet is barely visible for $\tau_{\text {evolve }} \simeq 2 \mathrm{~ms}$ (image a), which is $1.7 \mathrm{~ms}$ before $t_{\text {collapse }}$. The images also show how the number of condensate atoms decreases with time. The time from the application of $a_{\text {quench }}$ until the acquisition of the images was fixed at $5.2 \mathrm{~ms}$.

FIG. 6: Quantitative jet measurements. a. The number of atoms in the jets versus $\tau_{\text {evolve }}$ for the conditions of Fig. 1 b. b. The spike density inferred from the kinetic energies of the jets. The bars indicate the range of shot-to-shot variability. For the analysis, we assumed the jets were disk shaped since the magnetic trap is axially symmetric. The images were taken perpendicular to the axial trap axis, viewing the disks edge-on. The jets expanded with $v \simeq 1 \mathrm{~mm} / \mathrm{s}$, which corresponds to a kinetic energy of $\sim 6 \mathrm{nK}$ and a radial prequench Gaussian rms width of $\sim 0.5 \mu \mathrm{m}$. Since the axial size was below our resolution limit, we could not measure the axial expansion rate. For estimating the spike density, we assumed an axial width equal to the harmonic oscillator length. The atom density in the spikes decreased for larger values of $\left|a_{\text {collapse }}\right|$, and was half as large for $a_{\text {collapse }}=-100 a_{0}$ as for $-30 a_{0}$. 


\section{Donley, Fig. 1}
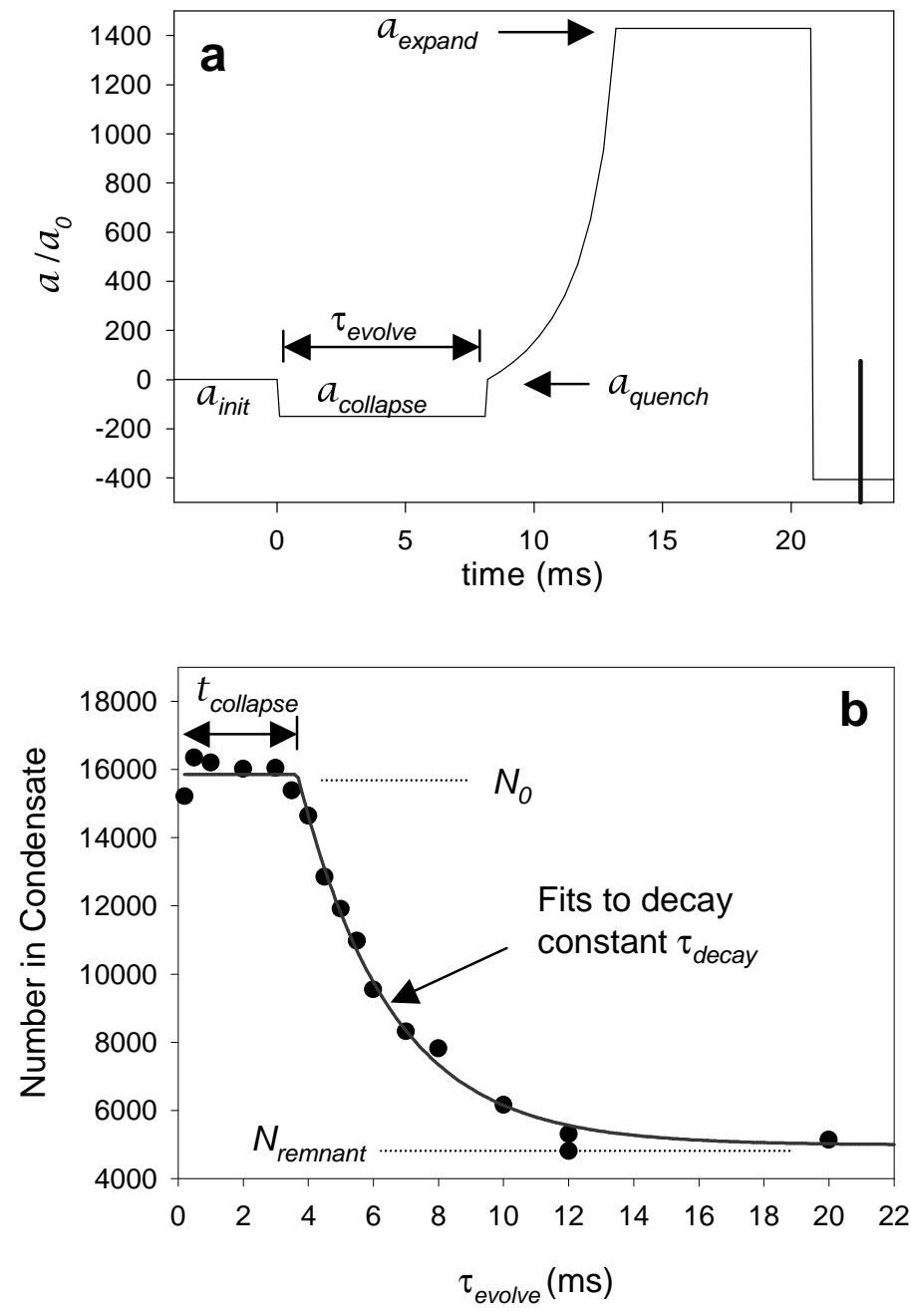
Donley, Fig. 2

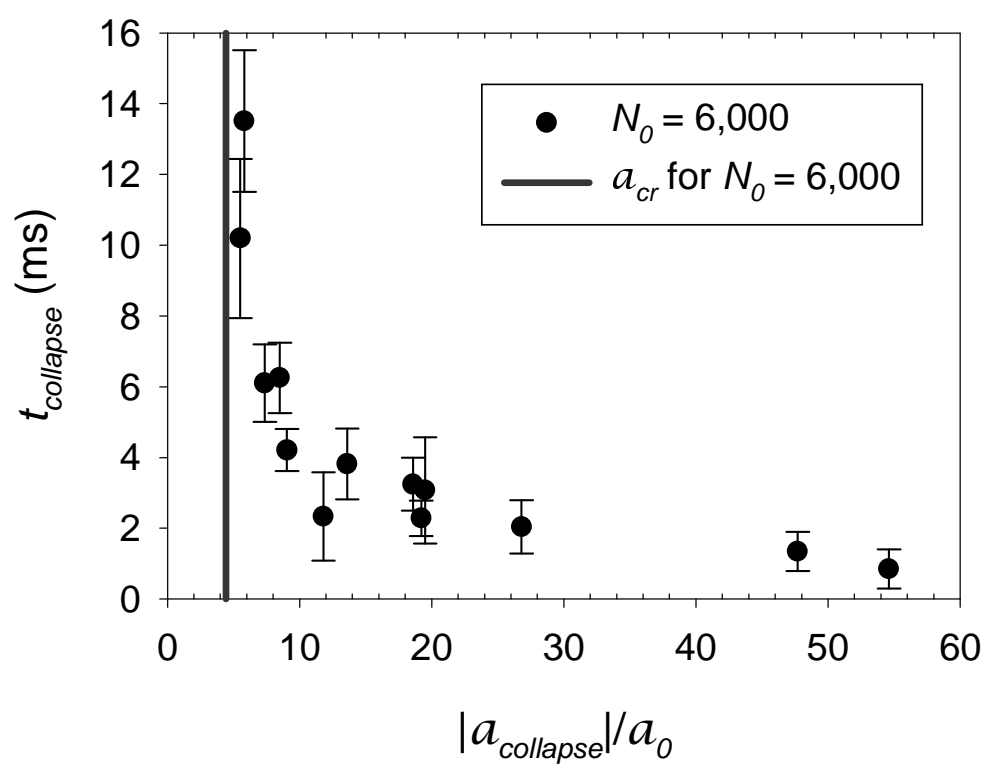




\section{Donley, Fig. 3}

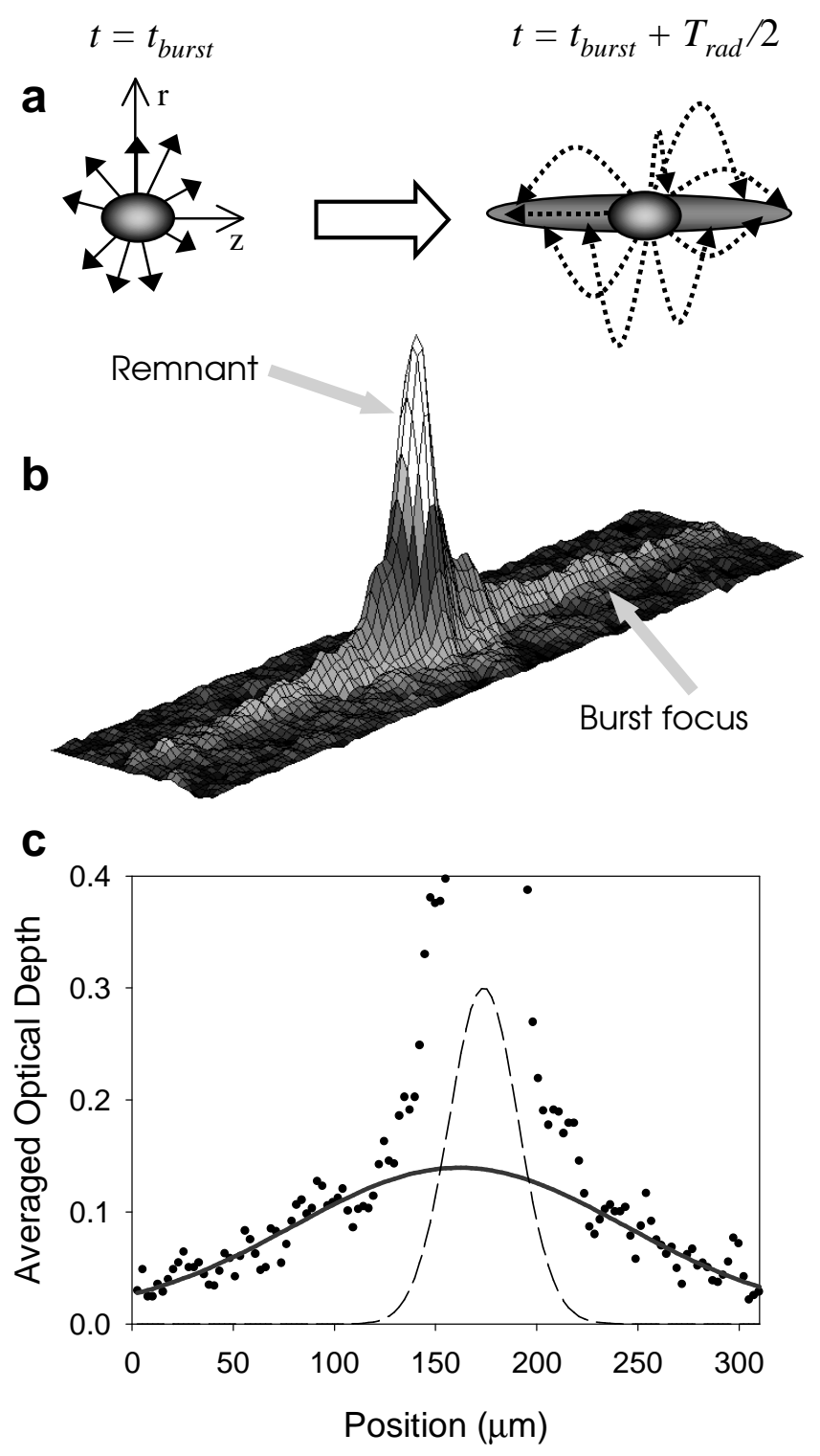




\section{Donley, Fig. 4}
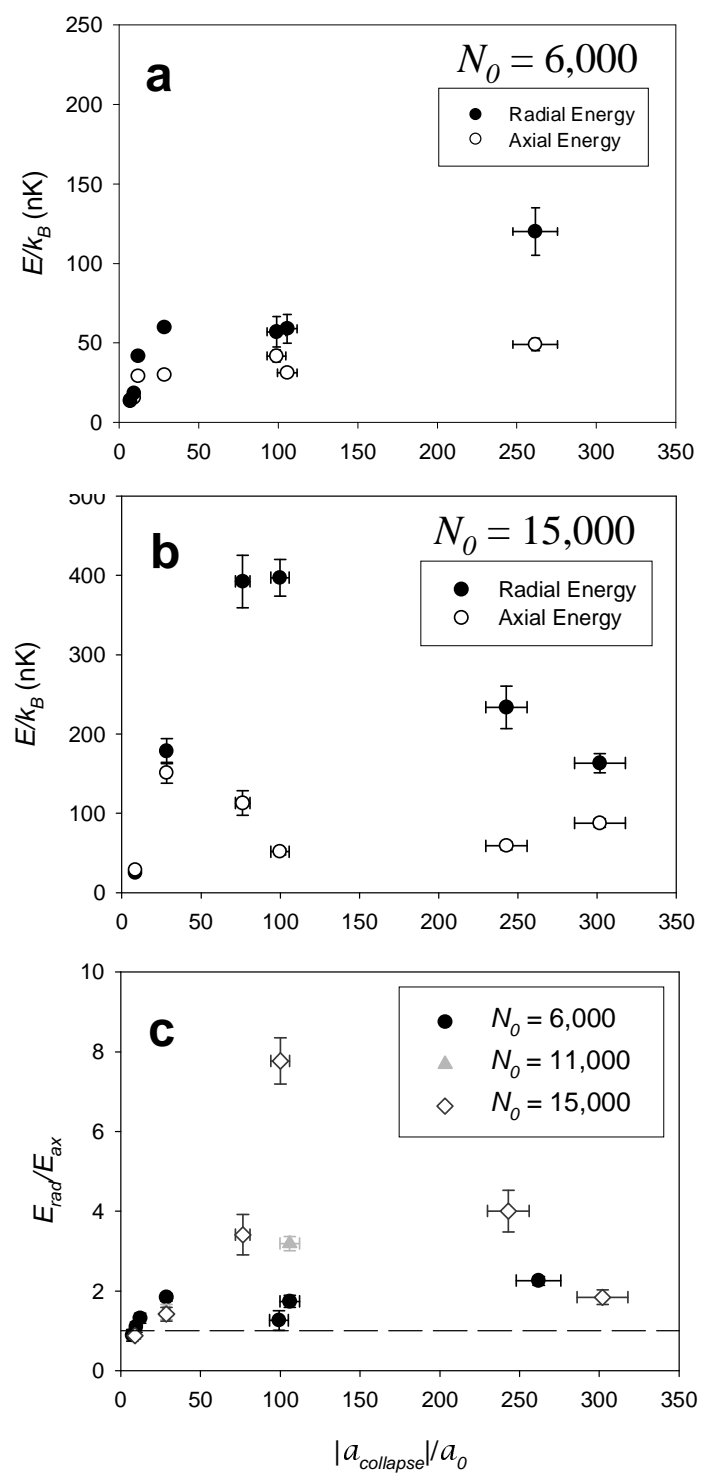
Donley, Fig. 5
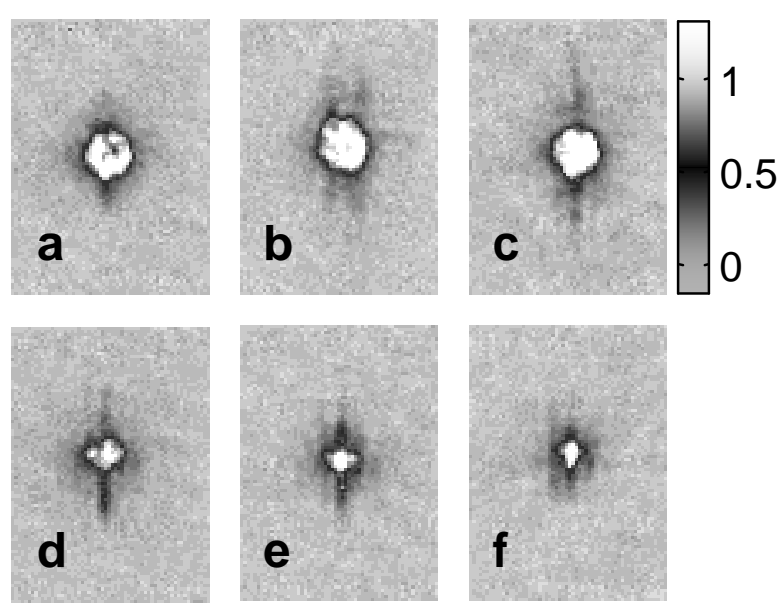
Donley, Fig. 6
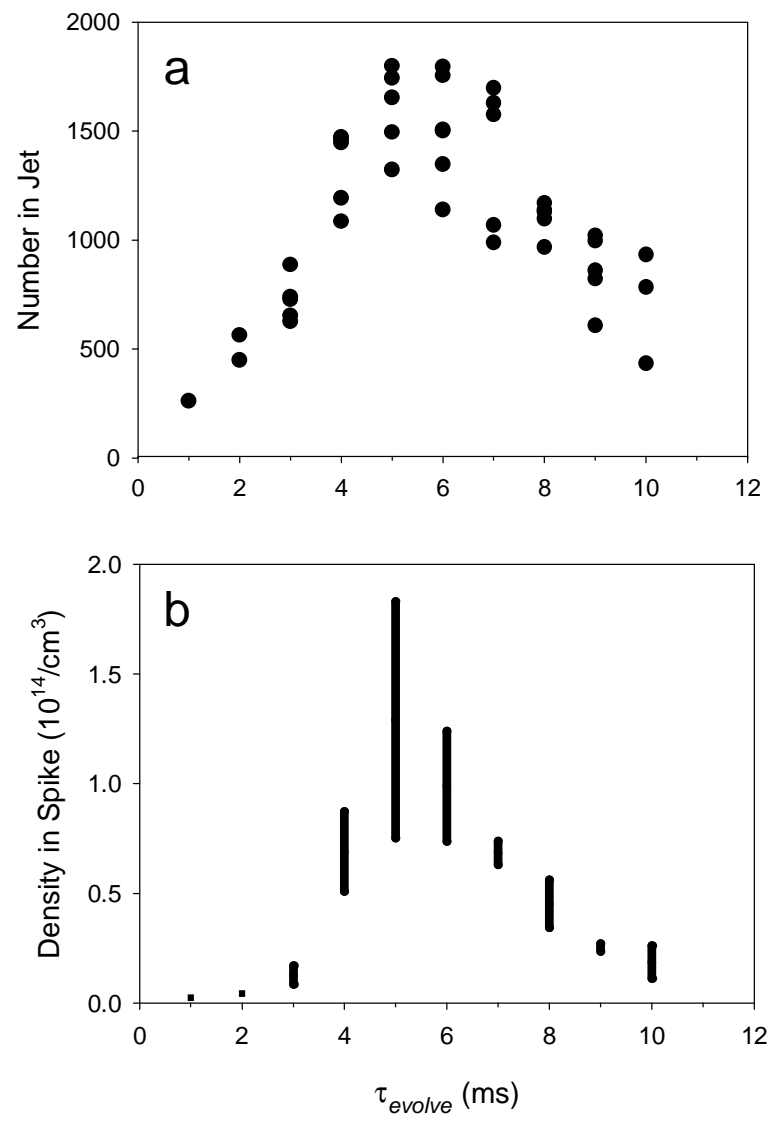